PROCEEDINGS OF THE

AMERICAN MATHEMATICAL SOCIETY

Volume 128, Number 10, Pages 2899-2901

S 0002-9939(00)05446-0

Article electronically published on February 28, 2000

\title{
NEVANLINNA FUNCTIONS AS QUOTIENTS
}

\author{
EVGUENI DOUBTSOV
}

(Communicated by Steven R. Bell)

\begin{abstract}
Let $f$ be a holomorphic function in the unit ball. Then $f$ is a Nevanlinna function if and only if there exist Smirnov functions $f_{+}, f_{-}$such that $f=f_{+} / f_{-}$and $f_{-}$has no zeros in the ball.
\end{abstract}

Let $B=B_{n}$ be the open unit ball in $\mathbb{C}^{n}$ and $S=\partial B$ be the unit sphere. If $n=1$, then $\mathbb{D}=B_{1}$ is the open unit disc in $\mathbb{C}$.

The Nevanlinna class $N(B)$ is the set of all holomorphic functions $f$ on $B$ such that

$$
\sup _{0<r<1} \int_{S} \log ^{+}\left|f_{r}\right| d \sigma<+\infty,
$$

where $\sigma$ is the normalized Lebesgue measure on $S, f_{r}(\zeta)=f(r \zeta)$, and $x^{+}=$ $\max (x, 0)$.

The Smirnov class $N^{+}(B)$ is the space of all functions $f \in N(B)$ such that the family $\left\{\log ^{+}\left|f_{r}\right|\right\}_{0<r<1}$ is uniformly integrable on $S$. Namely, for every $\varepsilon>0$, there exists $\delta>0$ such that

$$
\int_{\Delta} \log ^{+}\left|f_{r}\right| d \sigma<\varepsilon
$$

for all $0<r<1$ and all sets $\Delta \subset S$ such that $\sigma(\Delta)<\delta$.

Let $f$ be a holomorphic function on the disc. The classical factorization theorem implies that $f \in N(\mathbb{D})$ if and only if $f=f_{+} / f_{-}$, where $f_{+}$and $f_{-}$are holomorphic and bounded by 1 on $\mathbb{D}$ and $f_{-}$has no zeros on $\mathbb{D}$. In particular, the functions $f$ and $f_{+}$have the same zero sets.

Now, assume $n \geq 2$. Then there are many ways to say that the factorization theorem does not hold in the ball $B_{n}$. For example, there exist many functions $f \in N(B), f \not \equiv 0$, with the following property: If $f_{+} \in H^{\infty}(B)$ satisfies $f_{+} / f$ is holomorphic, then $f_{+} \equiv 0$. Hence, the above characterization does not hold for $n \geq 2$. Moreover, the zero sets of the Hardy classes $H^{p}(B)$ are all different (see [5]). One more negative result in this direction is the following nonfactorization theorem: The set $\left\{g h: g, h \in H^{2}(B)\right\}$ is a set of first category in $H^{1}(B)$ (see [3]).

However, in the present note we obtain the following description of the Nevanlinna class in the ball.

Received by the editors October 29, 1998.

2000 Mathematics Subject Classification. Primary 32A35.

Key words and phrases. Nevanlinna class, Smirnov class.

(C)2000 American Mathematical Society 
Theorem 1. Let $f$ be a holomorphic function on $B_{n}, n \geq 2$. Then $f \in N(B)$ if and only if there exist $f_{+}, f_{-} \in N^{+}(B)$ such that $f=f_{+} / f_{-}$and $f_{-}(z) \neq 0$ for all $z \in B$.

Proof. If $f_{+}, f_{-} \in N^{+}(B)$ and $f_{+} / f_{-}$is a holomorphic function, then $f_{+} / f_{-}$is a Nevanlinna function since

$$
\sup _{\frac{1}{2} \leq r<1} \int_{S}|\log |\left(f_{-}\right)_{r}|| d \sigma<\infty .
$$

So assume $f \in N(B)$. The Henkin-Skoda theorem provides a function $f_{0} \in$ $N^{+}(B)$ which has the same zeros (see 44 and 6]). More precisely, $f=f_{0} F$, where $F \in H(B)$ and $F(z) \neq 0$ for all $z \in B$. Without loss of generality $F(0) \in \mathbb{R}$.

Observe that $F \in N(B)$. Therefore $u=\log |F|$ is a pluriharmonic function and $\sup _{0<r<1} \int_{S}\left|u_{r}\right| d \sigma<\infty$. Hence, there exists a real measure $\mu$ on the sphere such that $u$ is the Poisson integral $P[\mu]$. Let $\mu=\mu_{s}+\mu_{a}$ be the Lebesgue decomposition of $\mu$ (here $\mu_{s} \perp \sigma$ and $\mu_{a} \ll \sigma$ ). Also, let $\mu_{s}=\mu_{+}-\mu_{-}, \mu_{ \pm} \geq 0$, be the Jordan decomposition of $\mu_{s}$. We have $\mu_{+} \ll \mu_{s}$; thus $\mu_{+}$is the singular part of a measure $\nu$ such that the Poisson integral $P[\nu]$ is a pluriharmonic function in the ball ([2], Corollary 2.7). In other words, there exists a function $g \in L^{1}(\sigma)$ such that $P\left[g \sigma-\mu_{+}\right]$is pluriharmonic. Denote by $F_{-}$the holomorphic function such that Re $F_{-}=P\left[g \sigma-\mu_{+}\right]$and $F_{-}(0) \in \mathbb{R}$. Respectively, the Poisson integral of the measure $\mu_{a}+g \sigma-\mu_{-}=\mu-\left(\mu_{+}-g \sigma\right)$ is a pluriharmonic function. So let $F_{+}$be the holomorphic function such that $\operatorname{Re} F_{+}=P\left[\mu_{a}+g \sigma-\mu_{-}\right]$and $F_{+}(0) \in \mathbb{R}$.

Clearly, the definitions of $F_{-}$and $F_{+}$yield the identity $F=\exp F_{+} \exp \left(-F_{-}\right)$. Put $f_{-}=\exp F_{-}$. Then $\log \left|\left(f_{-}\right)_{r}\right|=P_{r}\left[g \sigma-\mu_{+}\right] \leq P_{r}[g \sigma]$ since $\mu_{+}$is a nonnegative measure. So $\log ^{+}\left|\left(f_{-}\right)_{r}\right| \leq P_{r}\left[g^{+} \sigma\right]$, hence, the family $\left\{\log ^{+}\left|\left(f_{-}\right)_{r}\right|\right\}_{0<r<1}$ is uniformly integrable. In other words $f_{-} \in N^{+}(B)$.

Analogously $\exp F_{+} \in N^{+}(B)$, and we put $f_{+}=f_{0} \exp F_{+}$.

Observe that even a nonvanishing Nevanlinna function is not necessarily a quotient $f_{+} / f_{-}$, where $f_{ \pm} \in H^{\infty}(B)$ and $f_{-}$has no zeros in the ball. Moreover, one has the following negative example.

Proposition 2. There exists $f \in N(B)$ such that $f(z) \neq 0$ for all $z \in B$ and $f$ cannot be represented as $f_{+} / f_{-}$, where $f_{+} \in N^{+}(B), f_{-} \in H^{\infty}(B)$ and $f_{-}$has no zeros in the ball.

Proof. Fix a point $\zeta \in S$. Let $m$ be the normalized Lebesgue measure on the circle $\mathbb{T}_{\zeta}=\{\lambda \zeta: \lambda \in \mathbb{T}\} \subset S$. Choose a strictly positive lower semicontinuous function $g \in L^{1}(\sigma)$ such that

$$
\sup _{0<r<1} \int_{S} P_{r}[m] g d \sigma=+\infty
$$

Since $g$ is lower semicontinuous, there exists a singular positive measure $\mu$ on $S$ such that $P[g \sigma-\mu]=\operatorname{Re} F$ for certain $F \in H(B)$ (see [1], Chapter 5, 4.2). Put $f=\exp (-F) \in N(B)$.

Now, suppose $f=f_{+} / f_{-}$, where $f_{ \pm}$are nonvanishing, $f_{+} \in N^{+}(B)$, and $f_{-} \in H^{\infty}(B)$. Since $f_{ \pm}$are zero-free, there exist measures $\nu_{ \pm} \in M(S)$ such that $-\log \left|f_{ \pm}\right|=P\left[\nu_{ \pm}\right]$. Without loss of generality, let $\log \left|f_{-}\right| \leq 0$, in other words $\nu_{-} \geq 0$. Let $\left(\nu_{ \pm}\right)_{s}$ be the singular parts of $\nu_{ \pm}$; then $\mu=\left(\nu_{-}\right)_{s}-\left(\nu_{+}\right)_{s}$. Recall that 
$f_{+} \in N^{+}(B)$, so $\left(\nu_{+}\right)_{s} \geq 0$. Thus $\nu_{-} \geq\left(\nu_{-}\right)_{s} \geq \mu$. Therefore $g \sigma \leq g \sigma+\nu_{-}-\mu$ and the Poisson integral $u=P\left[(g \sigma-\mu)+\nu_{-}\right]$is pluriharmonic, hence

$$
u(0)=\sup _{0<r<1} \int_{S} u_{r} d m \geq \sup _{0<r<1} \int_{S} P_{r}[g \sigma] d m=+\infty .
$$

A contradiction.

\section{REFERENCES}

1. A.B. Aleksandrov, Function theory in the ball, in: Several Complex Variables II (eds. G.M. Khenkin and A.G. Vitushkin) Encyclopaedia Math. Sci., vol. 8, Springer-Verlag, Berlin, 1994, 107-178. MR 95e:32001

2. E. Doubtsov, Henkin measures, Riesz products and singular sets, Ann. Inst. Fourier (Grenoble) 48 (1998), 699-728. CMP 98:17

3. M.S. Gowda, Nonfactorization theorems in weighted Bergman and Hardy spaces on the unit ball of $\mathbb{C}^{n}(n>1)$, Trans. Amer. Math. Soc. 277 (1983), 203-212. MR 84i:32005

4. G.M. Henkin, H. Lewy's equation and analysis on a pseudoconvex manifold, II, Math. USSRSb. 102 (144) (1977), 63-94. MR 57:12907

5. W. Rudin, Zeros of holomorphic functions in balls, Indag. Math. 38 (1976), 57-65. MR 52:14347

6. H. Skoda, Valeurs au bord pour les solutions de l'opérateur d", et caractérisation des zéros des fonctions de la classe de Nevanlinna, Bull. Soc. Math. France 104 (1976), 225-299. MR 56:8913

Department of Mathematics, Michigan State University, East Lansing, Michigan 48824

E-mail address: dubtsov@math.msu.edu 